Maltepe Journal of Mathematics

ISSN:2667-7660, URL:HTTP://DERGIPARK.ORG.TR/TR/PUB/MJM VOLUME II IsSUE 2

(2020), Pages 61-75. Doi: 10.47087/MJM.723886

\title{
SOFT SEPARATION AXIOMS AND SOFT PRODUCT OF SOFT TOPOLOGICAL SPACES
}

\author{
RAMKUMAR SOLAI*, AND VINOTH SUBBIAH** \\ *GOVERNMENT POLYTECHNIC COLLEGE, GANDHARVAKOTTAI, PUDUKKOTTAI \\ DISTRICT - 613301, TAMILNADU, INDIA. \\ ORCID:HTTPS://ORCID.ORG/0000-0001-5011-774X \\ **VIGNAN'S UNIVERSITY, VADLAMUDI, GUNDUR - 522 213, ANDHRA PRADHESH, \\ INDIA. ORCID: HTTPS://ORCID.ORG/0000-0001-8621-8032
}

\begin{abstract}
In this article, we deal with the soft separation axioms using soft points on soft topological space and discuss the characterizations and properties of them. We extend these separation axioms to the soft product of soft topological spaces. Also we provide correct examples for the wrong examples example:1, example:2 and example:3 given in article [8].
\end{abstract}

For the vagueness and uncertinity of real life problems, there are several mathematical tools such as fuzzy sets, intuitionistic fuzzy sets, rough sets, vague sets etc. There is one more mathematical tool named soft sets which was introduced by Molodsov [12] in 1999. After that it was developed and used in decision making problems by Maji et. al in [10] and 11. Aktas and Cagman [1] introduced the applications of soft set theory in algebraic structures in 2007. Kharral and Ahmad [9] introduced and discussed several properties of soft mappings. Shabir and Naz [16] investigated soft seperation axioms defined for crisp points in 2011. Hussain and Ahmad 7 investigate the properties of soft interior, soft closure and soft boundary in 2011. Aygunoglu and Aygun 2] in 2012 generalize Alexander subbase theorem and Tychonoff theorem to the soft topological spaces by defining and using the product of soft topological spaces. Nazmul and Samanta [13] studied the neighbourhood properties of soft topological spaces in 2013. There are several articles related to the properties of soft topological spaces and soft mappings on soft topological spaces. Some of them are [4, 6, [14, 17, 19, 20, 21]. Four different types of sepereation axioms were defined and discussed in [5, [8, [16] and [18]. Singh and Noorie [17] derives the relation among these four types of $T_{i}, i=1,2,3,4$ spaces in 2017.

In the second section of this article, we give some basic definitions and preliminaries of soft topological spaces.

In the third section of this article, we deal with the soft separation axioms using soft points and discuss about the characterizations and properties of them. In fact

2010 Mathematics Subject Classification. Primary: 54D10 ; Secondaries: 54A40, 54A05 .

Key words and phrases. Soft toplogical spaces; Soft separation axioms; Soft product space.

(C)2020 Maltepe Journal of Mathematics.

Submitted on April 20th,2020. Published on October 30th, 2020.

Communicated by Pratulananda DAS. 
these separation axioms are stronger than other separation axioms. We extend these separation axioms to the product of soft topological spaces. Also we provide correct examples for the wrong examples Example:1, Example:2 and Example:3 given in article [8]

Throughout this paper, $X$ is the universe set, $E$ is a set of parameters and $\mathcal{P}(X)$ is the set of all subsets of $X$.

\section{Preliminaries}

Definition 1.1. 12 A mapping $F: E \rightarrow \mathcal{P}(X)$ is called a soft set and is denoted by $(F, E)$. The family of all soft sets over $X$ is denoted as $S S(X, E)$

Definition 1.2. [12 Let $(F, E)$ and $(G, E)$ be two soft sets over $X$. Then $(F, E)$ is a soft subset of $(G, E)$ written as $(F, E) \tilde{\subseteq}(G, E)$, if $F(e) \subseteq G(e)$, for all $e \in E$. Also the soft sets $(F, E)$ and $(G, E)$ are equal written as $(F, E) \tilde{=}(G, E)$, if $(F, E) \tilde{\tilde{\subseteq}}(G, E)$ and $(G, E) \tilde{\tilde{\subseteq}}(F, E)$.

Definition 1.3. 12] Let $\left\{\left(F_{i}, E\right): i \in I\right\} \tilde{\subseteq} S S(X, E)$, where $I$ is an arbitrary index set. Then

(1) the soft union of $\left\{\left(F_{i}, E\right): i \in I\right\}$ is the soft set $(F, E)$, where $F$ is the mapping defined as $F(e)=\cup\left\{F_{i}(e): i \in I\right\}$, for every $e \in E$ and is denoted as $(F, E)=\tilde{\cup}\left\{\left(F_{i}, E\right): i \in I\right\}$.

(2) the soft intersection of $\left\{\left(F_{i}, E\right): i \in I\right\}$ is the soft set $(F, E)$, where $F$ is the mapping defined as $F(e)=\cap\left\{F_{i}(e): i \in I\right\}$, for every $e \in E$ and is denoted as $(F, E)=\tilde{\cap}\left\{\left(F_{i}, E\right): i \in I\right\}$.

Definition 1.4. 21] Let $(F, E)$ be a soft set over $X$. Then the soft relative complement $F^{c}$ of $(F, E)$ is the mapping from $E$ to $\mathcal{P}(X)$ defined by $F^{c}(e)=$ $X-F(e)$ for every $e \in E$ and is denoted as $(F, E)^{c}$ or $\left(F^{c}, E\right)$.

Definition 1.5. [12] Let $(F, E)$ be a soft set over $X$. Then

(1) $(F, E)$ is called as null soft set, if $F(e)=\phi$, for every $e \in E$. We simply write it as $\tilde{\phi}$.

(2) $(F, E)$ is called as absolute soft set, if $F(e)=X$, for every $e \in E$. We simply write it as $\tilde{X}$.

Definition 1.6. ([16, 21] ) Let $\tau \subseteq S S(X, E)$. Then $\tau$ is a soft topology on $X$ if it satifies the following three conditions

(1) $\tilde{\phi}, \tilde{X} \in \tau$.

(2) The soft union of any number of soft sets in $\tau$ is in $\tau$.

(3) The soft intersection of finite number of soft sets in $\tau$ is in $\tau$.

This soft topological space over $X$ is written as $(X, \tau, E)$ and the members of $\tau$ are called as soft open sets in $X$. Also the soft complement of soft open sets are called soft closed sets.

Definition 1.7. 21] The soft set $(F, E)$ over $X$ is called as a soft point in $X$, denoted by $x_{e}$, if $F\left(e^{\prime}\right)= \begin{cases}\{x\} & \text { if } e^{\prime}=e \\ \phi & \text { if } e^{\prime} \in E-\{e\}\end{cases}$ 
Definition 1.8. 2] Let $(X, \tau, E)$ be a soft topological space. A subcollection $\mathcal{B}$ of $\tau$ is said to be a base for $\tau$ if every member of $\tau$ can be expressed as a union of members of $\tau$.

Definition 1.9. 2 Let $(X, \tau, E)$ be a soft topological space. A subcollection $\mathcal{S}$ of $\tau$ is said to be a subbase for $\tau$ if the family of all finite intersetions of members of $\mathcal{S}$ forms a base for $\tau$.

Definition 1.10. 21 A soft set $(G, E)$ in a soft topological space $(X, \tau, E)$ is known as a soft neighbourhood of a soft set $(F, E)$ if there exists a soft open set $(H, E)$ such that $(F, E) \tilde{\simeq}(H, E) \tilde{\subseteq}(G, E)$.

Definition 1.11. 16

Let $(F, E)$ be a soft set in a soft topological space $(X, \tau, E)$. Then the soft closure of $(F, E)$ is denoted as $C l(F, E)$ and defined as $C l(F, E)=\tilde{\cap}\{(G, E)$ : $(G, E) \tilde{\in} \tau^{c}$ and $\left.(G, E) \tilde{\supseteq}(F, E)\right\}$.

Definition 1.12. [16] Let $Y$ be a nonempty soft subset of a soft topological space $(X, \tau, E)$. Then $\tau_{Y}=\left\{(F, E) \tilde{\cap} E_{Y}:(F, E) \tilde{\in} \tau\right\}$ is called a soft relative topology on $Y$ and $\left(Y, \tau_{Y}, E\right)$ is called a soft subspace of $(X, \tau, E)$, where $E_{Y}: E \rightarrow \mathcal{P}(Y)$ such that $E_{Y}(e)=Y$, for every $e \in E$.

Proposition 1.1. 16 Let $\left(Y, \tau_{Y}, E\right)$ be a soft subspace of a soft topological space $(X, \tau, E)$ and $(F, A)$ be a soft set over $Y$. Then $(F, A)$ is a soft open set in $Y$ if and only if $(F, E)=(G, E) \tilde{\cap} E_{Y}$, for some $(G, E) \tilde{\in} \tau$.

Theorem 1.2. 21] A soft set $(F, E)$ is soft open set if and only if $(G, E)$ is a soft neighbourhood of a soft set $(F, E)$, for each soft set $(F, E)$ contained in $(G, E)$.

Proposition 1.3. [16] Let $(X, \tau, E)$ be a soft topological space over $X$. Then the collection $\tau_{e}=\{F(e):(F, E) \tilde{\in} \tau\}$ defines a topology on $X$.

Proposition 1.4. 16] Let $(X, \tau, E)$ be a soft topological space over $X$ and $Y \subseteq X$. Then $\left(Y, \tau_{Y_{e}}\right)$ is a subspace of $\left(X, \tau_{e}\right)$.

Definition 1.13. 3] Let $\left(F, E_{1}\right) \tilde{\epsilon} S S\left(X_{1}, E_{1}\right)$ and $\left(G, E_{2}\right) \tilde{\in} S S\left(X_{2}, E_{2}\right)$. Then the cartesian product $\left(F, E_{1}\right) \times\left(G, E_{2}\right)$ is defined by $(F \times G)_{\left(E_{1} \times E_{2}\right)}$, where $(F \times G)_{\left(E_{1} \times E_{2}\right)}\left(e_{1_{i}}, e_{2_{j}}\right)=F\left(e_{1_{i}}\right) \times G\left(e_{2_{j}}\right), \forall\left(e_{1_{i}}, e_{2_{j}}\right) \in E_{1} \times E_{2}$.

Definition 1.14. 2] The soft mappings $\left(p_{q}\right)_{i}, i \in\{1,2\}$ is called soft projection mappings from $X_{1} \times X_{2}$ to $X_{i}$ defined by $\left(p_{q}\right)_{i}\left((F, E)_{1} \times(F, E)_{2}\right)=\left(p_{q}\right)_{i}\left(\left(F_{1} \times\right.\right.$ $\left.\left.F_{2}\right)_{\left(E_{1} \times E_{2}\right)}\right)=p_{i}\left(F_{1} \times F_{2}\right)_{q_{i}\left(E_{1} \times E_{2}\right)}=(F, E)_{i}$, where $(F, E)_{1} \in S S\left(X_{1}, E_{1}\right)$, $(F, E)_{2} \in S S\left(X_{2}, E_{2}\right)$ and $p_{i}: X_{1} \times X_{2} \rightarrow X_{i}, q_{i}: E_{1} \times E_{2} \rightarrow E_{i}$ are projection mappings in classical meaning.

Definition 1.15. 2] Let $\left\{\left(\phi_{\psi}\right)_{i}: S(X, E) \rightarrow\left(Y_{i}, \tau_{i}\right)\right\}_{i \in \Delta}$ be a family of soft mappings where $\left\{\left(Y_{i}, \tau_{i}\right)\right\}_{i \in \Delta}$ be a family of soft topological spaces. Then the topology $\tau$ generated from the subbase $\left\{\left(\phi_{\psi}\right)_{i}^{-1}((F, E)):(F, E) \in \tau_{i}, i \in \Delta\right\}$ is called the initial soft topology induced by the family of soft mappings $\left\{\left(\phi_{\psi}\right)_{i}\right\}_{i \in \Delta}$.

Definition 1.16. 2] Let $\left\{\left(X_{i}, \tau_{i}\right)\right\}_{i \in \Delta}$ be a family of soft topological spaces. Then the initial soft topology on $X\left(=\prod_{i \in \Delta} X_{i}\right)$ generated by the family $\left\{\left(p_{q}\right)_{i}\right\}_{i \in \Delta}$ is called soft product topology on $X$, where $\left(p_{q}\right)_{i}$ are the soft projection mapping from $X$ to $X_{i}$. 
Theorem 1.5. [9] Let $X$ and $Y$ be crisp sets, $F_{A},\left(F_{A}\right)_{i} \in S S(X, E)$ and $G_{B},\left(G_{B}\right)_{i} \in S S(Y, K)$, where $i \in \Delta$, an index set.Then

(1) If $\left(F_{A}\right)_{1} \tilde{\subseteq}\left(F_{A}\right)_{2}$, then $\Phi_{\psi}\left(\left(F_{A}\right)_{1}\right) \tilde{\subseteq} \Phi_{\psi}\left(\left(F_{A}\right)_{2}\right)$.

(2) If $\left(G_{B}\right)_{1} \tilde{\subseteq}\left(G_{B}\right)_{2}$, then $\Phi_{\psi}^{-1}\left(\left(G_{B}\right)_{1}\right) \tilde{\subseteq} \Phi_{\psi}^{-1}\left(\left(G_{B}\right)_{2}\right)$.

(3) $\left(F_{A}\right) \tilde{\subseteq} \Phi_{\psi}^{-1}\left(\Phi_{\psi}\left(F_{A}\right)\right)$, the equality holds if $\Phi_{\psi}$ is injective.

(4) $\Phi_{\psi}\left(\Phi_{\psi}^{-1}\left(F_{A}\right)\right) \tilde{\simeq}\left(F_{A}\right)$, the equality holds if $\Phi_{\psi}$ is surjective.

(5) $\Phi_{\psi}\left(\cup_{i \in \Delta}^{\tilde{U}}\left(F_{A}\right)_{i}\right)=\underset{i \in \Delta}{\tilde{U}} \Phi_{\psi}\left(\left(F_{A}\right)_{i}\right)$.

(6) $\Phi_{\psi}\left(\bigcap_{i \in \Delta}\left(F_{A}\right)_{i}\right) \tilde{\subseteq} \bigcap_{i \in \Delta} \Phi_{\psi}\left(\left(F_{A}\right)_{i}\right)$.

(7) $\Phi_{\psi}^{-1}\left(\cap_{i \in \Delta}\left(G_{B}\right)_{i}\right)=\bigcap_{i \in \Delta} \Phi_{\psi}^{-1}\left(\left(G_{B}\right)_{i}\right)$.

(8) $\Phi_{\psi}^{-1}\left(\bigcap_{i \in \Delta}\left(G_{B}\right)_{i}\right)=\bigcap_{i \in \Delta} \Phi_{\psi}^{-1}\left(\left(G_{B}\right)_{i}\right)$.

(9) $\Phi_{\psi}^{-1}\left(E_{Y}\right)=E_{X}$ and $\Phi_{\psi}^{-1}\left(\phi_{Y}\right)=\phi_{X}$.

(10) $\Phi_{\psi}\left(E_{X}\right)=E_{Y}$ if $\Phi_{\psi}$ is surjective.

(11) $\Phi_{\psi}\left(\phi_{x}\right)=\phi_{Y}$.

\section{Soft SEPARATion AXioms and product soft topological SPACES}

Definition 2.1. 8] A soft topological space $(X, \tau, E)$ is said to be a soft $T_{0}$-space if for every pair of soft points $x_{e_{1}}, y_{e_{2}}$ such that $x_{e_{1}} \neq y_{e_{2}}$, there exists $(F, E) \in \tau$ such that $x_{e_{1}} \tilde{\in}(F, E), y_{e_{2}} \tilde{\notin}(F, E)$ or there exists $(G, E) \in \tau$ such that $y_{e_{2}} \tilde{\in}(G, E)$, $x_{e_{1}} \tilde{\notin}(G, E)$.

Definition 2.2. 8] A soft topological space $(X, \tau, E)$ is said to be a soft $T_{1}$-space if every pair of soft points $x_{e_{1}}, y_{e_{2}}$, such that $x_{e_{1}} \neq y_{e_{2}}$ there exist $(F, E),(G, E) \in \tau$ such that $x_{e_{1}} \tilde{\epsilon}(F, E), y_{e_{2}} \tilde{\notin}(F, E)$ and $x_{e_{1}} \tilde{\notin}(G, E), y_{e_{2}} \tilde{\epsilon}(G, E)$.

Example 2.1. Example for $T_{0}$-space.

Let $X=\{x, y\}, E=\left\{e_{1}, e_{2}\right\}$ and $\tau=\left\{\tilde{\phi}, \tilde{X},\left(F_{1}, E\right),\left(F_{2}, E\right),\left(F_{3}, E\right),\left(F_{4}, E\right)\right\}$ where

$F_{1}(e)=\left\{\begin{array}{ll}\{x\} & \text { if } e=e_{1} \\ \{y\} & \text { if } e=e_{2}\end{array}, F_{2}(e)=\left\{\begin{array}{ll}\{x\} & \text { if } e=e_{1} \\ \{x\} & \text { if } e=e_{2}\end{array}\right.\right.$,

$F_{3}(e)=\left\{\begin{array}{ll}\{x\} & \text { if } e=e_{1} \\ X & \text { if } e=e_{2}\end{array}, F_{4}(e)=\left\{\begin{array}{ll}\{x\} & \text { if } e=e_{1} \\ \phi & \text { if } e=e_{2}\end{array}\right.\right.$,

For the soft points $x_{e_{1}}, y_{e_{1}}$, there is a soft open set $\left(F_{1}, E\right) \in \tau$ with $x_{e_{1}} \tilde{\in}\left(F_{1}, E\right)$ and $y_{e_{1}} \tilde{\notin}\left(F_{1}, E\right)$. For the soft points $x_{e_{2}}, y_{e_{2}}$, there is a $\left(F_{1}, E\right) \in \tau$ with $x_{e_{2}} \tilde{\notin}\left(F_{1}, E\right)$ and $y_{e_{2}} \tilde{\in}\left(F_{1}, E\right)$. For the soft points $x_{e_{1}}, y_{e_{2}}$, there is $a\left(F_{2}, E\right) \in \tau$ with $x_{e_{1}} \tilde{\in}$ $\left(F_{2}, E\right)$ and $y_{e_{2}} \notin\left(F_{2}, E\right)$. For the soft points $x_{e_{2}}, y_{e_{1}}$, there is a $\left(F_{2}, E\right) \in \tau$ with $x_{e_{2}} \tilde{\in}\left(F_{2}, E\right)$ and $y_{e_{1}} \tilde{\notin}\left(F_{2}, E\right)$. For the soft points $x_{e_{1}}, x_{e_{2}}$, there is a $\left(F_{1}, E\right) \in \tau$ with $x_{e_{1}} \tilde{\in}\left(F_{1}, E\right)$ and $x_{e_{2}} \tilde{\notin}\left(F_{1}, E\right)$. For the soft points $y_{e_{1}}, y_{e_{2}}$, there is a $\left(F_{1}, E\right) \in \tau$ with $y_{e_{1}} \tilde{\notin}\left(F_{1}, E\right)$ and $y_{e_{2}} \tilde{\in}\left(F_{1}, E\right)$. Thus $(X, \tau, E)$ is a soft $T_{0}$-space.

Example 2.2. Let $X=\mathbf{Z}$, the set of all integers and $E=\mathbf{N}$, the set of all natural numbers. Define a soft topology on $X$ as $\tau=\left\{(F, E)^{c}: F\left(e_{i}\right)\right.$ is finite for each $\left.e_{i} \in E\right\} \cup\{\tilde{\phi}\}$.

(1) Clearly $\tilde{\phi} \in \tau$ and $\tilde{X} \in \tau$. 
(2) If $\left(F_{\alpha}, E\right) \in \tau$ for some $\alpha \in \Delta$, where $\Delta$ is some index set, then $F_{\alpha}^{c}\left(e_{i}\right)$ is finite for each $e_{i} \in E$. Now $\cap F_{\alpha}^{c}\left(e_{i}\right)=\left(\cup F_{\alpha}\left(e_{i}\right)\right)^{c}$ is finite for each $e_{i} \in E$. So that $\cup\left(F_{\alpha}, E\right) \in \tau$.

(3) If $\left(F_{1}, E\right),\left(F_{2}, E\right) \in \tau, F_{1}^{c}\left(e_{i}\right)$ and $F_{2}^{c}\left(e_{i}\right)$ are finite for each $e_{i} \in E$. Now $F_{1}^{c}\left(e_{i}\right) \cup F_{2}^{c}\left(e_{i}\right)=\left(F_{1}\left(e_{i}\right) \cap F_{2}\left(e_{i}\right)\right)^{c}=\left(\left(F_{1} \cap F_{2}\right)\left(e_{i}\right)\right)^{c}=\left(F_{1} \cap F_{2}\right)^{c}\left(e_{i}\right)$ is finite for each $e_{i} \in E$. So that $\left(F_{1}, E\right) \cap\left(F_{2}, E\right) \in \tau$.

Thus $(X, \tau, E)$ is a soft topological space. For any two distinct soft points $x_{e_{i}}$ and $y_{e_{j}}, x_{e_{i}}^{c}$ and $y_{e_{j}}^{c}$ are soft open sets such that $x_{e_{i}} \in y_{e_{j}}^{c}, y_{e_{j}} \notin y_{e_{j}}^{c}$ and $x_{e_{i}} \notin x_{e_{i}}^{c}$, $y_{e_{j}} \in x_{e_{i}}^{c}$. Thus $(X, \tau, E)$ is a soft $T_{1}$ space.

Theorem 2.1. Every soft $T_{1}$-space is a soft $T_{0}$-space.

Proof. Proof is straight forward

Theorem 2.2. Let $(X, \tau, E)$ be a soft topological space. Then $(X, \tau, E)$ is a soft $T_{0}$ space if and only if for any two distinct soft points $x_{e_{i}}$ and $y_{e_{j}}$, there is soft closed set $(H, E)$ such that $x_{e_{i}} \tilde{\in}(H, E), y_{e_{j}} \tilde{\notin}(H, E)$ or there is soft closed set $(K, E)$ such that $x_{e_{i}} \tilde{\notin}(K, E), y_{e_{j}} \tilde{\epsilon}(K, E)$.

Proof. Let us consider two distinct soft points $x_{e_{i}}$ and $y_{e_{j}}$. Since $(X, \tau, E)$ is a soft $T_{0}$ space, there is soft open set $(F, E)$ such that $x_{e_{i}} \tilde{\in}(F, E), y_{e_{j}} \tilde{\notin}(F, E)$ or there is soft open set $(G, E)$ such that $x_{e_{i}} \tilde{\notin}(G, E), y_{e_{j}} \tilde{\in}(G, E)$. Let $(H, E)=$ $\left(G^{c}, E\right)$ and $(K, E)=\left(F^{c}, E\right)$. Then $(H, E)$ is a soft closed set such that $x_{e_{i}} \tilde{\epsilon}$ $(H, E), y_{e_{j}} \tilde{\notin}(H, E)$ or $(K, E)$ is a soft closed set such that $x_{e_{i}} \tilde{\notin}(K, E), y_{e_{j}} \tilde{\epsilon}$ $(K, E)$.

Conversely, for any two distinct soft points $x_{e_{i}}$ and $y_{e_{j}}$, there is a soft closed set $(H, E)$ such that $x_{e_{i}} \tilde{\in}(H, E), y_{e_{j}} \tilde{\notin}(H, E)$ or there is soft closed set $(K, E)$ such that $x_{e_{i}} \tilde{\notin}(K, E), y_{e_{j}} \tilde{\in}(K, E)$. Then $\left(H^{c}, E\right)$ is a soft open set such that $x_{e_{i}} \tilde{\notin}$ $\left(H^{c}, E\right), y_{e_{j}} \tilde{\in}\left(H^{c}, E\right)$ or $\left(K^{c}, E\right)$ is a soft open set such that $x_{e_{i}} \tilde{\in}\left(K^{c}, E\right), y_{e_{j}} \tilde{\notin}$ $\left(K^{c}, E\right)$. This proves that $(X, \tau, E)$ is a soft $T_{0}$ space.

Example: 1 given in the artice [8] for soft $T_{1}$ space which is not a soft $T_{0}$ space is wrong. Because it is not a soft $T_{0}$ space too.

Example 2.3. [8] $X=\left\{x_{1}, x_{2}\right\}, A=\left\{e_{1}, e_{2}\right\}$ and $\tau=\{\tilde{\phi}, \tilde{X},(F, A)\}$ where $F(e)=\left\{\begin{array}{ll}\left\{x_{1}\right\} & \text { if } e=e_{1} \\ \left\{x_{2}\right\} & \text { if } e=e_{2}\end{array}\right.$ This $(X, \tau, A)$ is verified as soft $T_{0}$ space in [8].

$$
\text { consider two soft points } e_{F}=\left\{\begin{array}{ll}
\left\{x_{2}\right\} & \text { if } e=e_{1} \\
\phi & \text { if } e=e_{2}
\end{array} \text { and } e_{G}=\left\{\begin{array}{ll}
\phi & \text { if } e=e_{1} \\
\left\{x_{1}\right\} & \text { if } e=e_{2}
\end{array},\right.\right.
$$

then there is no soft open set $(F, A)$ in $(X, \tau, A)$ such that $e_{F} \tilde{\in}(F, A)$ and $e_{G} \tilde{\notin}(F, A)$. Thus $(X, \tau, A)$ is not a soft $T_{0}$ space.

The following example will be a correct example for example:1 of [8]. It also shows that the converse of above theorem 2.1 is not true in general.

Example 2.4. Example for a soft $T_{0}$-space which is not a soft $T_{1}$-space.

Let $X=\{x, y\}, E=\left\{e_{1}, e_{2}\right\}$ and $\tau=\left\{\tilde{\phi}, \tilde{X},\left(F_{1}, E\right),\left(F_{2}, E\right),\left(F_{3}, E\right),\left(F_{4}, E\right)\right.$, $\left.\left(F_{5}, E\right)\right\}$ where

$F_{1}(e)=\left\{\begin{array}{ll}\{x\} & \text { if } e=e_{1} \\ \{y\} & \text { if } e=e_{2}\end{array}, F_{2}(e)=\left\{\begin{array}{ll}\{x\} & \text { if } e=e_{1} \\ \phi & \text { if } e=e_{2}\end{array}, F_{3}(e)=\left\{\begin{array}{ll}\phi & \text { if } e=e_{1} \\ \{x\} & \text { if } e=e_{2}\end{array}\right.\right.\right.$, 
$F_{4}(e)=\left\{\begin{array}{ll}\{x\} & \text { if } e=e_{1} \\ \{x\} & \text { if } e=e_{2}\end{array}, F_{5}(e)= \begin{cases}\{x\} & \text { if } e=e_{1} \\ X & \text { if } e=e_{2}\end{cases}\right.$

For the soft points $x_{e_{1}}, y_{e_{1}}$, there is a $\left(F_{2}, E\right) \in \tau$ with $x_{e_{1}} \tilde{\in}\left(F_{2}, E\right)$ and $y_{e_{1}} \tilde{\notin}$ $\left(F_{2}, E\right)$. For the soft points $x_{e_{2}}, y_{e_{2}}$, there is a $\left(F_{3}, E\right) \in \tau$ with $x_{e_{2}} \tilde{\in}\left(F_{3}, E\right)$ and $y_{e_{2}} \notin\left(F_{3}, E\right)$. For the soft points $x_{e_{1}}, y_{e_{2}}$, there is a $\left(F_{2}, E\right) \in \tau$ with $x_{e_{1}} \tilde{\in}\left(F_{2}, E\right)$ and $y_{e_{2}} \tilde{\notin}\left(F_{2}, E\right)$. For the soft points $x_{e_{2}}, y_{e_{1}}$, there is a $\left(F_{3}, E\right) \in \tau$ with $x_{e_{2}} \tilde{\epsilon}$ $\left(F_{3}, E\right)$ and $y_{e_{1}} \notin\left(F_{3}, E\right)$. For the soft points $x_{e_{1}}, x_{e_{2}}$, there is $a\left(F_{2}, E\right) \in \tau$ with $x_{e_{1}} \tilde{\in}\left(F_{2}, E\right)$ and $x_{e_{2}} \tilde{\notin}\left(F_{2}, E\right)$. For the soft points $y_{e_{1}}, y_{e_{2}}$, there is a $\left(F_{1}, E\right) \in$ $\tau$ with $y_{e_{2}} \tilde{\in}\left(F_{1}, E\right)$ and $y_{e_{1}} \tilde{\notin}\left(F_{1}, E\right)$. Thus $(X, \tau, E)$ is a soft $T_{0}$-space. But for the pair of soft points $y_{e_{1}}, y_{e_{2}}$, we dont have $(K, E) \in \tau$ such that $y_{e_{1}} \tilde{\in}(K, E)$ and $y_{e_{2}} \notin(K, E)$. Thus $(X, \tau, E)$ is not a soft $T_{1}$-space.

Theorem 2.3. (1) A subspace of a soft $T_{0}$-space is a soft $T_{0}$-space.

(2) A subspace of a soft $T_{1}$-space is a soft $T_{1}$-space

Proof. (1) Let $(X, \tau, E)$ be a soft $T_{0}$-space and $\left(Y, \tau_{Y}, E\right)$ be a soft subspace. Let $x_{e_{i}}, y_{e_{j}}$ be two soft points in $S S(Y, E)$. Then $x_{e_{i}}, y_{e_{j}} \tilde{\in} S S(X, E)$. Since $(X, \tau, E)$ is a soft $T_{0}$ space, there is a soft open set $(F, E)$ in $(X, \tau, E)$ such that $x_{e_{i}} \tilde{\in}(F, E), y_{e_{j}} \tilde{\notin}(F, E)$ or there is a soft open set $(G, E)$ in $(X, \tau, E)$ such that $y_{e_{j}} \tilde{\epsilon}(G, E), x_{e_{i}} \tilde{\notin}(G, E)$. Then $(F, E) \tilde{\cap} E_{Y}$ is a soft open set in $\left(Y, \tau_{Y}, E\right)$ such that $x_{e_{i}} \tilde{\in}(F, E) \tilde{\cap} E_{Y} y_{e_{j}} \tilde{\notin}(F, E) \tilde{\cap} E_{Y}$ or $(G, E) \tilde{\cap} E_{Y}$ is a soft open set in $\left(Y, \tau_{Y}, E\right)$ such that $y_{e_{j}} \tilde{\in}(G, E) \tilde{\cap} E_{Y}$, $x_{e_{i}} \tilde{\notin}(G, E) \tilde{\cap} E_{Y}$. Thus $(Y, \tau, E)$ is a soft $T_{0}$-space

(2) Proof is similar to (1)

Theorem 2.4. Let $(X, \tau, E)$ be a soft topological space. Then $(X, \tau, E)$ is a soft $T_{1}$ space if and only if for any soft points $x_{e_{i}}$ and $y_{e_{j}}$, there exist two soft closed sets $(H, E)$ and $(K, E)$ such that $x_{e_{i}} \tilde{\in}(H, E), y_{e_{j}} \tilde{\notin}(H, E), y_{e_{j}} \tilde{\in}(K, E)$ and $x_{e_{i}} \tilde{\notin}(K, E)$.

Proof. Proof is similar to the theorem 2.2

The following example shows that the product of soft $T_{0}$-spaces need not be a soft $T_{0}$-space

Definition 2.3. Let $\left\{\left(X_{i}, \tau_{i}, E_{i}\right): i \in I\right\}$ be a family of soft topological spaces and $\left(\prod X_{i}, \prod \tau_{i}, \prod E_{i}\right)$ be their product soft toplogical space. Then a soft point in $\left(\prod X_{i}, \prod \tau_{i}, \prod E_{i}\right)$ is denoted as $\mathbf{x}_{\mathbf{e}}$, where $\mathbf{x}=<x_{i}>_{i \in I}, x_{i} \in X_{i}$ and $\mathbf{e}=<$ $e_{i}>_{i \in I}, e_{i} \in E_{i}$.

Example 2.5. Let $X_{1}=\left\{x_{1}, y_{1}\right\}, E_{1}=\left\{e_{11}, e_{12}\right\}$ and $\tau_{1}=\left\{\tilde{\phi}, \tilde{X}_{1},\left(F_{1}, E_{1}\right)\right.$, $\left.\left(F_{2}, E_{1}\right),\left(F_{3}, E_{1}\right),\left(F_{4}, E_{1}\right),\left(F_{5}, E_{1}\right),\left(F_{6}, E_{1}\right),\left(F_{7}, E_{1}\right)\right\} . X_{2}=\left\{x_{2}, y_{2}\right\}, E_{2}=$ $\left\{e_{21}, e_{22}\right\}$ and $\tau_{2}=\left\{\tilde{\phi}, \tilde{X}_{2},\left(G_{1}, E_{2}\right),\left(G_{2}, E_{2}\right),\left(G_{3}, E_{2}\right),\left(G_{4}, E_{2}\right),\left(G_{5}, E_{2}\right),\left(G_{6}, E_{2}\right)\right.$, $\left.\left(G_{7}, E_{2}\right)\right\}$ where

$F_{1}(e)=\left\{\begin{array}{ll}\left\{x_{1}\right\} & \text { if } e=e_{11} \\ \phi & \text { if } e=e_{12}\end{array}, G_{1}(e)=\left\{\begin{array}{ll}\left\{x_{2}\right\} & \text { if } e=e_{21} \\ \phi & \text { if } e=e_{22}\end{array}, F_{2}(e)=\left\{\begin{array}{ll}\phi & \text { if } e=e_{11} \\ \left\{x_{1}\right\} & \text { if } e=e_{12}\end{array}\right.\right.\right.$,
$G_{2}(e)=\left\{\begin{array}{ll}\phi & \text { if } e=e_{21} \\ \left\{x_{2}\right\} & \text { if } e=e_{22}\end{array}, F_{3}(e)=\left\{\begin{array}{ll}\left\{x_{1}\right\} & \text { if } e=e_{11} \\ \left\{x_{1}\right\} & \text { if } e=e_{12}\end{array}, G_{3}(e)=\left\{\begin{array}{ll}\left\{x_{2}\right\} & \text { if } e=e_{21} \\ \left\{x_{2}\right\} & \text { if } e=e_{22}\end{array}\right.\right.\right.$, 
SOFT SEPARATION AXIOMS AND SOFT PRODUCT OF SOFT TOPOLOGICAL SPACES 67

$$
\begin{aligned}
& F_{4}(e)=\left\{\begin{array}{ll}
\left\{y_{1}\right\} & \text { if } e=e_{11} \\
\left\{x_{1}\right\} & \text { if } e=e_{12}
\end{array}, G_{4}(e)=\left\{\begin{array}{ll}
\left\{x_{2}\right\} & \text { if } e=e_{21} \\
\left\{y_{2}\right\} & \text { if } e=e_{22}
\end{array}, F_{5}(e)=\left\{\begin{array}{ll}
\left\{y_{1}\right\} & \text { if } e=e_{11} \\
\phi & \text { if } e=e_{12}
\end{array},\right.\right.\right. \\
& G_{5}(e)=\left\{\begin{array}{ll}
\phi & \text { if } e=e_{21} \\
\left\{y_{2}\right\} & \text { if } e=e_{22}
\end{array}, F_{6}(e)=\left\{\begin{array}{ll}
X_{1} & \text { if } e=e_{11} \\
\phi & \text { if } e=e_{12}
\end{array}, G_{6}(e)=\left\{\begin{array}{ll}
\phi & \text { if } e=e_{21} \\
X_{2} & \text { if } e=e_{22}
\end{array},\right.\right.\right. \\
& F_{7}(e)=\left\{\begin{array}{ll}
X_{1} & \text { if } e=e_{11} \\
\left\{x_{1}\right\} & \text { if } e=e_{12}
\end{array}, G_{7}(e)=\left\{\begin{array}{ll}
\left\{x_{2}\right\} & \text { if } e=e_{21} \\
X_{2} & \text { if } e=e_{22}
\end{array} .\right.\right.
\end{aligned}
$$

For the soft points $x_{1_{e_{11}}}, y_{1_{e_{11}}}$, there is a soft open set $\left(F_{1}, E_{1}\right) \in \tau_{1}$ with $x_{1_{e_{11}}} \tilde{\epsilon}$ $\left(F_{1}, E_{1}\right)$ and $y_{1_{e_{11}}} \tilde{\notin}\left(F_{1}, E_{1}\right)$. For the soft points $x_{1_{e_{11}}}, y_{1_{e_{12}}}$, there is a soft open set $\left(F_{1}, E_{1}\right) \in \tau_{1}$ with $x_{1_{e_{11}}} \tilde{\in}\left(F_{1}, E_{1}\right)$ and $y_{1_{e_{12}}} \tilde{\notin}\left(F_{1}, E_{1}\right)$. For the soft points $x_{1_{e_{12}}}$, $y_{1_{e_{11}}}$, there is $\left(F_{2}, E_{1}\right) \in \tau_{1}$ with $x_{1_{e_{12}}} \tilde{\in}\left(F_{2}, E_{1}\right)$ and $y_{1_{e_{11}}} \tilde{\notin}\left(F_{2}, E_{1}\right)$. For the soft points $x_{1_{e_{12}}}, y_{1_{e_{12}}}$, there is $\left(F_{2}, E_{1}\right) \in \tau_{1}$ with $x_{1_{e_{12}}} \tilde{\in}\left(F_{2}, E_{1}\right)$ and $y_{1_{e_{12}}} \tilde{\notin}\left(F_{2}, E_{1}\right)$. For the soft points $x_{1_{e_{11}}}, x_{1_{e_{12}}}$, there is a soft open set $\left(F_{1}, E_{1}\right) \in \tau_{1}$ with $x_{1_{e_{11}}} \tilde{\epsilon}$ $\left(F_{1}, E_{1}\right)$ and $x_{1_{e_{12}}} \tilde{\notin}\left(F_{1}, E_{1}\right)$. For the soft points $y_{1_{e_{11}}}, y_{1_{e_{12}}}$, there is a soft open set $\left(F_{5}, E_{1}\right) \in \tau_{1}$ with $y_{1_{e_{11}}} \tilde{\in}\left(F_{5}, E_{1}\right)$ and $y_{1_{e_{12}}} \notin\left(F_{5}, E_{1}\right)$. Thus $\left(X_{1}, \tau_{1}, E_{1}\right)$ is a soft $T_{0}$-space.

For the soft points $x_{2_{e_{21}}}, y_{2_{e_{21}}}$, there is a soft open set $\left(G_{1}, E_{2}\right) \in \tau_{2}$ with $x_{2_{e_{21}}} \tilde{\epsilon}$ $\left(G_{1}, E_{2}\right)$ and $y_{2_{e_{21}}} \tilde{\notin}\left(G_{1}, E_{2}\right)$. For the soft points $x_{2_{e_{21}}}, y_{2_{e_{22}}}$, there is a soft open set $\left(G_{1}, E_{2}\right) \in \tau_{2}$ with $x_{2_{e_{21}}} \tilde{\in}\left(G_{1}, E_{2}\right)$ and $y_{2_{e_{22}}} \tilde{\notin}\left(G_{1}, E_{2}\right)$. For the soft points $x_{2_{e_{22}}}, y_{2_{e_{21}}}$, there is a soft open set $\left(G_{2}, E_{2}\right) \in \tau_{2}$ with $x_{2_{e_{22}}} \tilde{\epsilon}\left(G_{2}, E_{2}\right)$ and $y_{2_{e_{21}}} \tilde{\notin}$ $\left(G_{2}, E_{2}\right)$. For the soft points $x_{2_{e_{22}}}, y_{2_{e_{22}}}$, there is a soft open set $\left(G_{2}, E_{2}\right) \in \tau_{2}$ with $x_{2_{e_{22}}} \tilde{\in}\left(G_{2}, E_{2}\right)$ and $y_{2_{e_{22}}} \tilde{\notin}\left(G_{2}, E_{2}\right)$. For the soft points $x_{2_{e_{21}}}, x_{2_{e_{22}}}$, there is a soft open set $\left(G_{1}, E_{2}\right) \in \tau_{2}$ with $x_{2_{e_{21}}} \tilde{\in}\left(G_{1}, E_{2}\right)$ and $x_{2_{e_{22}}} \tilde{\notin}\left(G_{1}, E_{2}\right)$. For the soft points $y_{2_{e_{21}}}, y_{2_{e_{22}}}$, there is a soft open set $\left(G_{4}, E_{2}\right) \in \tau_{2}$ with $y_{2_{e_{21}}} \tilde{\notin}\left(G_{4}, E_{2}\right)$ and $y_{2_{e_{22}}} \tilde{\in}\left(G_{4}, E_{2}\right)$. Thus $\left(X_{2}, \tau_{2}, E_{2}\right)$ is a soft $T_{0}$-space.

Now $E_{1} \times E_{2}=\left\{\left(e_{11}, e_{21}\right),\left(e_{11}, e_{22}\right),\left(e_{12}, e_{21}\right),\left(e_{12} e_{22}\right)\right\}$ and $\tau_{1} \times \tau_{2}=\{\tilde{\phi}$, $X_{1} \tilde{\times} X_{2},\left(F_{1} \times G_{1}, E_{1} \times E_{2}\right),\left(F_{1} \times G_{2}, E_{1} \times E_{2}\right),\left(F_{1} \times G_{3}, E_{1} \times E_{2}\right),\left(F_{1} \times G_{4}, E_{1} \times\right.$ $\left.E_{2}\right),\left(F_{1} \times G_{5}, E_{1} \times E_{2}\right),\left(F_{1} \times G_{6}, E_{1} \times E_{2}\right),\left(F_{1} \times G_{7}, E_{1} \times E_{2}\right),\left(F_{2} \times G_{1}, E_{1} \times E_{2}\right)$, $\left(F_{2} \times G_{2}, E_{1} \times E_{2}\right),\left(F_{2} \times G_{3}, E_{1} \times E_{2}\right),\left(F_{2} \times G_{4}, E_{1} \times E_{2}\right),\left(F_{2} \times G_{5}, E_{1} \times E_{2}\right)$, $\left(F_{2} \times G_{6}, E_{1} \times E_{2}\right),\left(F_{2} \times G_{7}, E_{1} \times E_{2}\right),\left(F_{3} \times G_{1}, E_{1} \times E_{2}\right),\left(F_{3} \times G_{2}, E_{1} \times E_{2}\right)$, $\left(F_{3} \times G_{3}, E_{1} \times E_{2}\right),\left(F_{3} \times G_{4}, E_{1} \times E_{2}\right),\left(F_{3} \times G_{5}, E_{1} \times E_{2}\right),\left(F_{3} \times G_{6}, E_{1} \times E_{2}\right)$, $\left(F_{3} \times G_{7}, E_{1} \times E_{2}\right),\left(F_{4} \times G_{1}, E_{1} \times E_{2}\right),\left(F_{4} \times G_{2}, E_{1} \times E_{2}\right),\left(F_{4} \times G_{3}, E_{1} \times E_{2}\right)$, $\left(F_{4} \times G_{4}, E_{1} \times E_{2}\right),\left(F_{4} \times G_{5}, E_{1} \times E_{2}\right),\left(F_{4} \times G_{6}, E_{1} \times E_{2}\right),\left(F_{4} \times G_{7}, E_{1} \times E_{2}\right)$, $\left(F_{5} \times G_{1}, E_{1} \times E_{2}\right),\left(F_{5} \times G_{2}, E_{1} \times E_{2}\right),\left(F_{5} \times G_{3}, E_{1} \times E_{2}\right),\left(F_{5} \times G_{4}, E_{1} \times E_{2}\right)$, $\left(F_{5} \times G_{5}, E_{1} \times E_{2}\right),\left(F_{5} \times G_{6}, E_{1} \times E_{2}\right),\left(F_{5} \times G_{7}, E_{1} \times E_{2}\right),\left(F_{6} \times G_{1}, E_{1} \times E_{2}\right)$, $\left(F_{6} \times G_{2}, E_{1} \times E_{2}\right),\left(F_{6} \times G_{3}, E_{1} \times E_{2}\right),\left(F_{6} \times G_{4}, E_{1} \times E_{2}\right),\left(F_{6} \times G_{5}, E_{1} \times E_{2}\right)$, $\left(F_{6} \times G_{6}, E_{1} \times E_{2}\right),\left(F_{6} \times G_{7}, E_{1} \times E_{2}\right),\left(F_{7} \times G_{1}, E_{1} \times E_{2}\right),\left(F_{7} \times G_{2}, E_{1} \times E_{2}\right)$, $\left(F_{7} \times G_{3}, E_{1} \times E_{2}\right),\left(F_{7} \times G_{4}, E_{1} \times E_{2}\right),\left(F_{7} \times G_{5}, E_{1} \times E_{2}\right),\left(F_{7} \times G_{6}, E_{1} \times E_{2}\right)$, $\left.\left(F_{7} \times G_{7}, E_{1} \times E_{2}\right)\right\}$. 
Suppose if the soft product of $\left(X_{1}, \tau_{1}, E_{1}\right)$ and $\left(X_{2}, \tau_{2}, E_{2}\right)$ is a soft $T_{0}$ space, then for any two distinct soft points $\left(x_{1}, y_{2}\right)_{\left(e_{11}, e_{21}\right)}= \begin{cases}\left\{\left(x_{1}, y_{2}\right)\right\} & \text { if } e=\left(e_{11}, e_{21}\right) \\ \phi & \text { if } e=\left(e_{11}, e_{22}\right) \\ \phi & \text { if } e=\left(e_{12}, e_{21}\right) \\ \phi & \text { if } e=\left(e_{12}, e_{22}\right)\end{cases}$ and $\left(y_{1}, y_{2}\right)_{\left(e_{11}, e_{21}\right)}=\left\{\begin{array}{ll}\left\{\left(y_{1}, y_{2}\right)\right\} & \text { if } e=\left(e_{11}, e_{21}\right) \\ \phi & \text { if } e=\left(e_{11}, e_{22}\right) \\ \phi & \text { if } e=\left(e_{12}, e_{21}\right) \\ \phi & \text { if } e=\left(e_{12}, e_{22}\right)\end{array}\right.$ there is a soft open set $\left(F_{m} \times G_{n}, E_{1} \times E_{2}\right)$ in $\tau_{1} \times \tau_{2}$ such that $\left(x_{1}, y_{2}\right)_{\left(e_{11}, e_{21}\right)} \tilde{\in}\left(F_{m} \times G_{n}, E_{1} \times E_{2}\right)$ and $\left(y_{1}, y_{2}\right)_{\left(e_{11}, e_{21}\right)} \tilde{\notin}\left(F_{m} \times G_{n}, E_{1} \times E_{2}\right)$, for some $m, n \in\{1,2,3 \ldots, 7\}$. Now $\left(p_{q}\right)_{2}\left(\left(x_{1}, y_{2}\right)_{\left(e_{11}, e_{21}\right)}\right) \tilde{\epsilon}\left(p_{q}\right)_{2}\left(\left(F_{m} \times G_{n}, E_{1} \times E_{2}\right)\right)$. That is $p_{2}\left(x_{1}, y_{2}\right)_{q_{2}\left(e_{11}, e_{21}\right)} \tilde{\epsilon}$ $p_{2}\left(F_{m} \times G_{n}\right)_{q_{2}\left(E_{1} \times E_{2}\right)}$. This implies $y_{2_{e_{21}}} \tilde{\in}\left(G_{n}, E_{2}\right)$, for some $m, n \in\{1,2,3 \ldots, 7\}$. Since $\left(p_{q}\right)_{2}$ is a soft projection mapping and $\left(F_{m} \times G_{n}, E_{1} \times E_{2}\right)$ is a soft open set in $X_{1} \times X_{2},\left(G_{n}, E_{2}\right)$ is a soft open set in $\left(X_{2}, \tau_{2}, E_{2}\right)$ containing $y_{2_{e_{21}}}$. But there is no soft open set $\left(G_{n}, E_{2}\right)$ in $\left(X_{2}, \tau_{2}, E_{2}\right)$ containing $y_{2_{e_{21}}}$, for any $n \in\{1,2,3 \ldots, 7\}$ and hence $\left(X_{1} \times X_{2}, \tau_{1} \times \tau_{2}, E_{1} \times E_{2}\right)$ is not a soft $T_{0}$ space.

Definition 2.4. Let $(X, \tau, E)$ be a soft topological space and $A=\left\{x_{e_{i}}: x_{e_{i}}\right.$ is a soft points of $(X, \tau, E)\}$.

(1) If the number of elements of the set $A$ is finite, then $(X, \tau, E)$ is called a finite soft topological space.

(2) If the number of elements of the set $A$ is countable, then $(X, \tau, E)$ is called a countable soft topological space.

Theorem 2.5. If $(X, \tau, E)$ is a finite soft $T_{1}$ space, then $(X, \tau, E)$ is a soft discrete space.

Proof. Let $x_{e_{i}}$ be a soft point, $x \in X$ and $e_{i} \in E .(X, \tau, E)$ is a soft $T_{1}$ space, for any soft point $y_{e_{j}} \neq x_{e_{i}}$, there is a soft open set $\left(F_{x_{j}}, E\right)$ such that $x_{e_{i}} \tilde{\epsilon}$ $\left(F_{x_{j}}, E\right)$ and $y_{e_{j}} \tilde{\notin}\left(F_{x_{j}}, E\right)$. Since $(X, \tau, E)$ is a finite soft topological space, $\tilde{y}_{e_{j} \neq x_{e_{i}}}^{\tilde{n}}\left(F_{x_{j}}, E\right)$ is a soft open set such that $\underset{y_{e_{j}} \neq x_{e_{i}}}{\tilde{n}}\left(F_{x_{j}}, E\right)=\left\{\begin{array}{ll}\{x\} & \text { if } e=e_{i} \\ \phi & \text { if } e \neq e_{i}\end{array}\right.$. Thus $x_{e_{i}}$ is soft open and hence $(X, \tau, E)$ is a soft discrete space.

Definition 2.5. Let $(X, \tau, E)$ be a soft topological space. Then the soft set $(F, E)$ is called a soft $G_{\delta}$ set if it is a countable intersection of soft open sets.

Theorem 2.6. If $(X, \tau, E)$ is a countable soft $T_{1}$ space and if every soft $G_{\delta}$ set is soft open in $(X, \tau, E)$, then $(X, \tau, E)$ is a soft discrete space.

Proof. Let $x_{e_{i}}$ be a soft point. Since $(X, \tau, E)$ is a soft $T_{1}$ space, for any soft point $y_{e_{j}} \neq x_{e_{i}}$, there is a soft open set $\left(F_{x_{j}}, E\right)$ such that $x_{e_{i}} \tilde{\in}\left(F_{x_{j}}, E\right)$ and $y_{e_{j}} \tilde{\notin}\left(F_{x_{j}}, E\right)$. Since every soft $G_{\delta}$ set is soft open and $(X, \tau, E)$ is a countable soft topological space, $\underset{y_{e_{j}} \neq x_{e_{i}}}{\tilde{n}}\left(F_{x_{j}}, E\right)$ is a soft open set such that $\underset{y_{e_{j}} \neq x_{e_{i}}}{\tilde{n}}\left(F_{x_{j}}, E\right)=$ $\left\{\begin{array}{ll}\{x\} & \text { if } e=e_{i} \\ \phi & \text { if } e \neq e_{i}\end{array}\right.$. Thus $x_{e_{i}}$ is soft open and hence $(X, \tau, E)$ is a soft discrete space. 
SOFT SEPARATION AXIOMS AND SOFT PRODUCT OF SOFT TOPOLOGICAL SPACES 69

Theorem 2.7. Product of soft $T_{1}$-spaces is a soft $T_{1}$-space

Proof. Let $\left\{\left(X_{i}, \tau_{i}, E_{i}\right): i \in I\right\}$ be a family of soft topological spaces and $\left(\prod X_{i}, \prod \tau_{i}, \prod E_{i}\right)$ be their product soft toplogical space. Suppose $\mathbf{x}_{\mathbf{e}}$ and $\mathbf{y}_{\mathbf{f}}$ be two distinct soft points, where $\mathbf{x}=<x_{i}>_{i \in I}, \mathbf{y}=<y_{i}>_{i \in I} \quad x_{i}, y_{i} \in X_{i}$ and $\mathbf{e}=<e_{i}>_{i \in I}, \mathbf{f}=<f_{i}>_{i \in I}, e_{i}, f_{i} \in E_{i}$. Then there exists atleast one $\beta \in I$ such that $x_{\beta} \neq y_{\beta}$ or there exist $e_{i_{k}}, e_{i_{m}} \in E_{i}$ such that $e_{i_{k}} \neq e_{i_{m}}$.

Case: 1

If $x_{\beta} \neq y_{\beta},\left(p_{q}\right)_{\beta}\left(\mathbf{x}_{\mathbf{e}}\right)=\left(p_{\beta_{q_{\beta}}}\right)\left(\mathbf{x}_{\mathbf{e}}\right)=p_{\beta}(\mathbf{x})_{q_{\beta}(\mathbf{e})}=x_{\beta_{e_{\beta}}}$ and $\left(p_{q}\right)_{\beta}\left(\mathbf{y}_{\mathbf{f}}\right)=\left(p_{\beta_{q_{\beta}}}\right)\left(\mathbf{y}_{\mathbf{f}}\right)=$ $p_{\beta}(\mathbf{y})_{q_{\beta}(\mathbf{f})}=y_{\beta_{f_{\beta}}}$. Since $X_{\beta}$ is a soft $T_{1}$ space, there exist soft open sets $\left(F_{\beta}, E_{\beta}\right)$ and $\left(G_{\beta}, E_{\beta}\right)$ such that $x_{\beta_{e_{\beta}}} \tilde{\epsilon}\left(F_{\beta}, E_{\beta}\right), y_{\beta_{f_{\beta}}} \tilde{\notin}\left(F_{\beta}, E_{\beta}\right)$ and $y_{\beta_{f_{\beta}}} \tilde{\epsilon}\left(G_{\beta}, E_{\beta}\right), x_{\beta_{e_{\beta}}}$ $\tilde{\notin}\left(G_{\beta}, E_{\beta}\right)$. Then the soft subbasic members $\left(p_{q}\right)_{\beta}^{-1}\left(F_{\beta}, E_{\beta}\right)$ and $\left(p_{q}\right)_{\beta}^{-1}\left(G_{\beta}, E_{\beta}\right)$ are the soft open sets containing $\mathbf{x}_{\mathbf{e}}$ and $\mathbf{y}_{\mathbf{f}}$ respectively. Suppose if $\mathbf{y}_{\mathbf{f}} \tilde{\in}\left(p_{q}\right)_{\beta}^{-1}\left(F_{\beta}, E_{\beta}\right)$, then $p_{\beta}(\mathbf{y})_{q_{\beta}(f)}=\left(p_{q}\right)_{\beta}\left(\mathbf{y}_{\mathbf{f}}\right) \tilde{\epsilon}\left(p_{q}\right)_{\beta}\left(\left(p_{q}\right)_{\beta}^{-1}\left(F_{\beta}, E_{\beta}\right)\right)$. That is $y_{\beta_{f_{\beta}}} \tilde{\in}\left(F_{\beta}, E_{\beta}\right)$ which is a contradiction. Similarly we can prove $\mathbf{x}_{\mathbf{e}} \tilde{\notin}\left(p_{q}\right)_{\beta}^{-1}\left(G_{\beta}, E_{\beta}\right)$. Thus $\left(p_{q}\right)_{\beta}^{-1}\left(F_{\beta}, E_{\beta}\right)$ and $\left(p_{q}\right)_{\beta}^{-1}\left(G_{\beta}, E_{\beta}\right)$ are the soft open sets such that $\mathbf{x}_{\mathbf{e}} \tilde{\in}\left(p_{q}\right)_{\beta}^{-1}\left(F_{\beta}, E_{\beta}\right), \mathbf{y}_{\mathbf{f}} \tilde{\notin}\left(p_{q}\right)_{\beta}^{-1}$ $\left(F_{\beta}, E_{\beta}\right)$ and $\mathbf{y}_{\mathbf{f}} \tilde{\in}\left(p_{q}\right)_{\beta}^{-1}\left(G_{\beta}, E_{\beta}\right), \mathbf{x}_{\mathbf{e}} \tilde{\notin}\left(p_{q}\right)_{\beta}^{-1}\left(G_{\beta}, E_{\beta}\right)$.

\section{Case: 2}

If $e_{i_{k}} \neq e_{i_{m}}$, there are soft open sets $\left(F_{i_{k}}, E_{i}\right)$ and $\left(F_{i_{m}}, E_{i}\right)$ in $\left(X_{i}, \tau_{i}, E_{i}\right)$ such that $x_{e_{i_{k}}} \tilde{\in}\left(F_{i_{k}}, E_{i}\right), x_{e_{i_{m}}}\left(=y_{e_{i_{m}}}\right) \tilde{\notin}\left(F_{i_{k}}, E_{i}\right)$ and $x_{e_{i_{m}}} \tilde{\in}\left(F_{i_{m}}, E_{i}\right), x_{e_{i_{k}}} \tilde{\notin}\left(F_{i_{m}}, E_{i}\right)$. Then $\left(p_{q}\right)_{i}^{-1}\left(F_{i_{k}}, E_{i}\right)$ and $\left(p_{q}\right)_{i}^{-1}\left(F_{i_{m}}, E_{i}\right)$ are soft open sets such that $\mathbf{x}_{\mathbf{e}} \tilde{\epsilon}$ $\left(p_{q}\right)_{i}^{-1}\left(F_{i_{k}}, E_{i}\right)$ and $\mathbf{y}_{\mathbf{f}} \tilde{\in}\left(p_{q}\right)_{i}^{-1}\left(F_{i_{m}}, E_{i}\right)$. We can prove $\mathbf{y}_{\mathbf{f}} \tilde{\notin}\left(p_{q}\right)_{i}^{-1}\left(F_{i_{k}}, E_{i}\right)$ and $\mathbf{x}_{\mathbf{e}} \notin\left(p_{q}\right)_{i}^{-1}\left(F_{i_{m}}, E_{i}\right)$ as we proved in case:1. This completes the proof.

Theorem 2.8. Let $(X, \tau, E)$ be a soft topological space. Then the following are equivalent.

(1) $(X, \tau, E)$ is a soft $\tau_{1}$-space

(2) $x_{e_{i}}=\tilde{\cap}\left\{(G, E):(G, E) \in \tau\right.$ and $\left.x_{e_{i}} \tilde{\in}(G, E)\right\}$

(3) $x_{e_{i}}=\tilde{\cap}\left\{(F, E):(F, E) \in \tau^{c}\right.$ and $\left.x_{e_{i}} \tilde{\in}(F, E)\right\}$

Proof. (i) $\Rightarrow$ (ii). Clearly $x_{e_{i}} \tilde{\subseteq} \tilde{\cap}\left\{(G, E):(G, E) \in \tau\right.$ and $\left.x_{e_{i}} \tilde{\in}(G, E)\right\}$. Suppose if $y_{e_{j}} \tilde{\in} \tilde{\cap}\left\{(G, E):(G, E) \in \tau\right.$ and $\left.x_{e_{i}} \tilde{\in}(G, E)\right\}$ such that $x_{e_{i}} \neq y_{e_{j}}$. Then $x \neq y$ or $e_{i} \neq e_{j}$. In either cases, by our assumption, there is a soft open set $(G, E)$ such that $x_{e_{i}} \tilde{\epsilon}(G, E)$ and $y_{e_{j}} \tilde{\notin}(G, E)$.So $y_{e_{j}} \tilde{\notin} \tilde{\cap}\left\{(G, E):(G, E) \tilde{\in} \tau\right.$ and $\left.x_{e_{i}} \tilde{\in}(G, E)\right\}$. Thus $x_{e_{i}}=\tilde{\cap}\left\{(G, E):(G, E) \in \tau\right.$ and $\left.x_{e_{i}} \tilde{\in}(G, E)\right\}$.

$($ ii $) \Rightarrow($ iii $)$. Clearly $x_{e_{i}} \tilde{\subseteq} \tilde{\cap}\left\{(F, E):(F, E) \in \tau^{c}\right.$ and $\left.x_{e_{i}} \tilde{\in}(F, E)\right\}$. Let $y_{e_{j}} \tilde{\in} \tilde{\cap}\{(F, E)$ $:(F, E) \in \tau^{c}$ and $\left.x_{e_{i}} \tilde{\in}(F, E)\right\}$ such that $x_{e_{i}} \neq y_{e_{j}}$. By (ii), there exists $(G, E) \in \tau$ such that $y_{e_{j}} \tilde{\epsilon}(G, E)$ and $x_{e_{i}} \tilde{\notin}(G, E)$. Now $(G, E)^{c} \in \tau^{c}$ and $y_{e_{j}} \tilde{\notin}(G, E)^{c}$ and $x_{e_{i}} \tilde{\in}(G, E)^{c}$ and hence $y_{e_{j}} \tilde{\notin} \tilde{\cap}\left\{(F, E):(F, E) \in \tau^{c}\right.$ and $\left.x_{e_{i}} \tilde{\in}(F, E)\right\}$. Thus $x_{e_{i}}=\tilde{\cap}$ $\left\{(F, E):(F, E) \in \tau^{c}\right.$ and $\left.x_{e_{i}} \tilde{\in}(F, E)\right\}$.

$($ iii $) \Rightarrow\left(\right.$ i). Let $x_{e_{i}}$ and $y_{e_{j}}$ be two distinct soft points. Then by (iii), $x_{e_{i}} \neq$ $y_{e_{j}}=\tilde{\cap}\left\{(F, E):(F, E) \in \tau^{c}\right.$ and $\left.y_{e_{j}} \tilde{\epsilon}(F, E)\right\}$. There is some soft closed set $\left(F_{1}, E\right)$ such that $y_{e_{j}} \tilde{\in}\left(F_{1}, E\right)$ and $x_{e_{i}} \tilde{\notin}\left(F_{1}, E\right)$. Then $\left(F_{1}, E\right)^{c}$ is a soft open set such that $x_{e_{i}} \tilde{\in}\left(F_{1}, E\right)^{c}$ and $y_{e_{j}} \tilde{\notin}\left(F_{1}, E\right)^{c}$. Similarly, from $y_{e_{j}} \neq x_{e_{i}}=\tilde{\cap}\{(F, E)$ : $(F, E) \in \tau^{c}$ and $\left.y_{e_{j}} \tilde{\in}(F, E)\right\}$, we can find another soft open set $\left(F_{2}, E\right)^{c}$ such that $x_{e_{i}} \tilde{\notin}\left(F_{2}, E\right)^{c}$ and $y_{e_{j}} \tilde{\in}\left(F_{2}, E\right)^{c}$. This proves that $(X, \tau, E)$ is a soft $\tau_{1}$-space. 
Remark. (1) From (iii) of theorem 2.8, it is clear that each soft point $x_{e_{i}}$ is a soft closed set in a soft $T_{1}$ space.

(2) Let $T_{i}=$ Number of elements in $F\left(e_{i}\right), i \in I$ an indexed set of $E$. If $T=$ $\sum_{i \in I} T_{i}$ is finite, then the soft set $(F, E)$ can be written as a finite union of soft points. Each soft point is a soft closed set, we have $(F, E)$ is a soft closed set.

(3) If $T=\sum_{i \in I} T_{i}$ is infinite, $(F, E)$ need not be a closed set. Following example shows this.

Example 2.6. Let $X$ be an infinite set and $E=\mathbf{N}$. Let $\tau=\left\{(F, E)^{c}:\left\{e_{i}\right.\right.$ : $\left.F\left(e_{i}\right) \neq \phi\right\}$ is finite $\} \cup\{\tilde{\phi}\}$.

(1) Clearly $\tilde{\phi} \in \tau$ and $\tilde{X} \in \tau$.

(2) If $\left(F_{\alpha_{i}}, E\right) \in \tau, \alpha_{i} \in I$, for some index set $I$, then $\left\{e_{j}: F_{\alpha_{i}}^{c}\left(e_{j}\right) \neq \phi\right\}$ is a finite set. Now $\left\{e_{j}:\left(\cup F_{\alpha_{i}}\right)^{c}\left(e_{j}\right) \neq \phi\right\}=\left\{e_{j}: \tilde{\cap} F_{\alpha_{i}}^{c}\left(e_{j}\right) \neq \phi\right\} \subseteq\left\{e_{j}:\right.$ $\left.F_{\alpha_{k}}^{c}\left(e_{j}\right) \neq \phi\right\}$, for all $\alpha_{k} \in I$. Since $\left\{e_{j}: F_{\alpha_{k}}^{c}\left(e_{j}\right) \neq \phi\right\}$ is a finite set, $\left\{e_{j}:\left(\cup F_{\alpha_{i}}\right)^{c}\left(e_{j}\right) \neq \phi\right\}$ is a finite set and hence $\tilde{\cup}\left(F_{\alpha_{i}}, E\right) \in \tau$.

(3) If $\left(F_{\alpha_{1}}, E\right)$ and $\left(F_{\alpha_{2}}, E\right) \in \tau$, then $\left\{e_{j}: F_{\alpha_{1}}^{c}\left(e_{j}\right) \neq \phi\right\}$ and $\left\{e_{j}: F_{\alpha_{2}}^{c}\left(e_{j}\right) \neq\right.$ $\phi\}$ are finite sets. Now $\left\{e_{j}:\left(F_{\alpha_{1}}^{c} \tilde{\cup} F_{\alpha_{2}}^{c}\right)\left(e_{j}\right) \neq \phi\right\}=\left\{e_{j}:\left(F_{\alpha_{1}} \tilde{\cap} F_{\alpha_{2}}\right)^{c}\left(e_{j}\right) \neq\right.$ $\phi\}$ is a finite set. Thus $\left(F_{\alpha_{1}}, E\right) \tilde{\cap}\left(F_{\alpha_{2}}, E\right) \in \tau$

Thus $(X, \tau, E)$ is a soft topological space. Let us take two distinct soft points $x_{e_{i}}$ and $y_{e_{j}}$. Then either $x \neq y$ or $e_{i} \neq e_{j}$. In either cases $x_{e_{i}}^{c}$ and $y_{e_{j}}^{c}$ are two soft open sets such that $x_{e_{i}} \tilde{\in} y_{e_{j}}^{c}, y_{e_{j}} \tilde{\notin} y_{e_{j}}^{c}$ and $x_{e_{i}} \tilde{\notin} x_{e_{i}}^{c}, y_{e_{j}} \tilde{\in} x_{e_{i}}^{c}$. This proves that $(X, \tau, E)$ is a soft $T_{1}$ space.

Let us consider a soft set $(G, E)$ such that $G\left(e_{i}\right)=\left\{\begin{array}{ll}\{x\} & \text { if } e_{i} \text { is even } \\ \phi & \text { if } e_{i} \text { is odd }\end{array}\right.$. Define $T\left(e_{i}\right)=\left\{\begin{array}{ll}1 & \text { if } e_{i} \text { is even } \\ 0 & \text { if } e_{i} \text { is odd }\end{array} . T=\sum T\left(e_{i}\right)=\infty\right.$, because $2 \mathbf{N}$ is an infinite set. Since $\left\{e_{j}: G\left(e_{j}\right) \neq \phi\right\}$ is not a finite set, $(G, E)$ is not a soft closed set.

Definition 2.6. 8] A soft topological space $(X, \tau, E)$ is said to be a soft $T_{2}$-space if for every pair of soft points $x_{e_{i}}$ and $y_{e_{j}}$ such that $x_{e_{i}} \neq y_{e_{j}}$ there exist soft open sets $(F, E)$ and $(G, E)$ such that $x_{e_{i}} \tilde{\in}(F, E), y_{e_{j}} \tilde{\in}(G, E)$ and $(F, E) \tilde{\cap}(G, E)=\tilde{\phi}$.

Example:2 given in the artice [8] for soft $T_{1}$ and soft $T_{2}$ space is wrong. Because it is neither soft $T_{1}$ nor soft $T_{2}$ space.

Example 2.7. 8] $X=\left\{x_{1}, x_{2}\right\}, A=\left\{e_{1}, e_{2}\right\}$ and $\tau=\left\{\tilde{\phi}, \tilde{X},\left(F_{1}, A\right),\left(F_{2}, A\right)\right.$, $\left.\left(F_{3}, A\right),\left(F_{4}, A\right)\right\}$ where

$F_{1}(e)=\left\{\begin{array}{ll}\left\{x_{2}\right\} & \text { if } e=e_{1} \\ \left\{x_{1}\right\} & \text { if } e=e_{2}\end{array}, F_{2}(e)=\left\{\begin{array}{ll}\left\{x_{1}\right\} & \text { if } e=e_{1} \\ \left\{x_{2}\right\} & \text { if } e=e_{2}\end{array} F_{3}(e)= \begin{cases}\left\{x_{1}\right\} & \text { if } e=e_{1} \\ \phi & \text { if } e=e_{2}\end{cases}\right.\right.$ $F_{4}(e)= \begin{cases}X & \text { if } e=e_{1} \\ \left\{x_{1}\right\} & \text { if } e=e_{2}\end{cases}$

This $(X, \tau, A)$ is verified as soft $T_{1}$ and soft $T_{2}$ spaces in [8]. consider two soft points $e_{F}=\left\{\begin{array}{ll}\left\{x_{1}\right\} & \text { if } e=e_{1} \\ \phi & \text { if } e=e_{2}\end{array}\right.$ and $e_{G}=\left\{\begin{array}{ll}\phi & \text { if } e=e_{1} \\ \left\{x_{2}\right\} & \text { if } e=e_{2}\end{array}\right.$, then there is no soft open set $\left(F_{i}, A\right), i \in\{1,2,3,4\}$ in $(X, \tau, A)$ such that $e_{G} \tilde{\in}\left(F_{i}, A\right)$ and $e_{F} \tilde{\notin}\left(F_{i}, A\right)$. Thus $(X, \tau, A)$ is not a soft $T_{1}$ space. 
Similarly, there is no two soft open sets $\left(F_{i}, A\right)\left(F_{j}, A\right), i, j \in\{1,2,3,4\}, i \neq j$ in $(X, \tau, A)$ such that $e_{F} \tilde{\in}\left(F_{i}, A\right), e_{G} \tilde{\in}\left(F_{j}, A\right)$ and $\left(F_{i}, A\right) \tilde{\cap}\left(F_{j}, A\right)=\tilde{\phi}$ Thus $(X, \tau, A)$ is not a soft $T_{2}$ space too.

Next the example:3 given in article [8] is wrong.

Example 2.8. [8] $X=\left\{x_{1}, x_{2}\right\}, A=\left\{e_{1}, e_{2}\right\}$ and $\tau=\left\{\tilde{\phi}, \tilde{X},\left(F_{1}, A\right),\left(F_{2}, A\right),\left(F_{3}, A\right)\right\}$ where

$F_{1}(e)=\left\{\begin{array}{ll}\left\{x_{1}\right\} & \text { if } e=e_{1} \\ \phi & \text { if } e=e_{2}\end{array}, F_{2}(e)=\left\{\begin{array}{ll}\phi & \text { if } e=e_{1} \\ \left\{x_{2}\right\} & \text { if } e=e_{2}\end{array} F_{3}(e)= \begin{cases}\left\{x_{1}\right\} & \text { if } e=e_{1} \\ \left\{x_{2}\right\} & \text { if } e=e_{2}\end{cases}\right.\right.$

This $(X, \tau, A)$ is verified as soft $T_{1}$ and soft $T_{0}$ spaces in $[8]$.

consider two soft points $e_{F}=\left\{\begin{array}{ll}\left\{x_{2}\right\} & \text { if } e=e_{1} \\ \phi & \text { if } e=e_{2}\end{array}\right.$ and $e_{G}=\left\{\begin{array}{ll}\phi & \text { if } e=e_{1} \\ \left\{x_{1}\right\} & \text { if } e=e_{2}\end{array}\right.$, then there is no soft open set $\left(F_{i}, A\right), i \in\{1,2,3\}$ in $(X, \tau, A)$ such that $e_{F} \tilde{\in}\left(F_{i}, A\right)$ and $e_{G} \tilde{\notin}\left(F_{i}, A\right)$. Thus $(X, \tau, A)$ is not a soft $T_{1}$ space. Also there is no soft open set $\left(F_{i}, A\right), i \in\{1,2,3\}$ in $(X, \tau, A)$ such that $e_{F} \tilde{\notin}\left(F_{i}, A\right)$ and $e_{G} \tilde{\in}\left(F_{i}, A\right)$. Hence $(X, \tau, A)$ is not a soft $T_{0}$ space too.

Correct example for soft $T_{1}$ spae which is not a soft $T_{2}$ space is given below.

Example 2.9. Consider a soft topological space $(X, \tau, E)$ discussed in Example: 2.6. It is a soft $T_{1}$ space.

Let $x_{e_{i}}$ and $y_{e_{j}}$ be two distinct soft points. Then either $x \neq y$ or $e_{i} \neq e_{j}$. Assume that there exists two soft open sets $(F, E)$ and $(G, E)$ such that $x_{e_{i}} \tilde{\in}(F, E)$ and $y_{e_{j}} \tilde{\in}(G, E)$. Since $(F, E)$ and $(G, E)$ are soft open sets, $\left\{e_{j}: F^{c}\left(e_{j}\right) \neq \phi\right\}$ and $\left\{e_{j}: G^{c}\left(e_{j}\right) \neq \phi\right\}$ are finite sets. Now $E-\left\{e_{j}:\left(F\left(e_{j}\right) \cap G\left(e_{j}\right)\right)^{c} \neq \phi\right\} \neq \phi$. For any $e_{k} \in E-\left\{e_{j}:\left(F\left(e_{j}\right) \cap G\left(e_{j}\right)\right)^{c} \neq \phi\right\}, F^{c}\left(e_{k}\right)=\phi$ and $G^{c}\left(e_{k}\right)=\phi$. That is $F\left(e_{k}\right) \cap G\left(e_{k}\right)=X$ and hence $(F, E) \tilde{\cap}(G, E) \neq \phi$. This proves that $(X, \tau, E)$ is not a soft $T_{2}$ space.

Theorem 2.9. Every soft $T_{2}$ space is a soft $T_{1}$ space

Proof. Proof is straight forward.

Theorem 2.10. Soft subspace of soft $T_{2}$-space is a soft $T_{2}$-space.

Proof. Let $(X, \tau, E)$ be a soft $T_{2}$-space and $\left(Y, \tau_{Y}, E\right)$ be a soft subspace. Let $x_{e_{i}}, y_{e_{j}}$ be two soft points in $(Y, \tau, E)$. Then $x_{e_{i}}, y_{e_{j}} \tilde{\in} S S(X, E)$. Since $(X, \tau, E)$ is a soft $T_{2}$ space, there exist two soft open sets $(F, E)$ and $(G, E)$ in $(X, \tau, E)$ such that $x_{e_{i}} \tilde{\in}(F, E), y_{e_{j}} \tilde{\in}(G, E)$ and $(F, E) \tilde{\cap}(G, E)=\tilde{\phi}$. Now $(F, E) \tilde{\cap} E_{Y}$ and $(G, E) \tilde{\cap} E_{Y}$ are soft open sets in $\left(Y, \tau_{Y}, E\right)$ such that $x_{e_{i}} \tilde{\in}(F, E) \tilde{\cap} E_{Y}, y_{e_{j}} \tilde{\epsilon}(G, E)$ $\tilde{\cap} E_{Y}$ and $\left((F, E) \tilde{\cap} E_{Y}\right) \tilde{\cap}\left((G, E) \tilde{\cap} E_{Y}\right) \tilde{\subseteq}(F, E) \tilde{\cap}(G, E)=\tilde{\phi}$. Thus $\left(Y, \tau_{Y}, E\right)$ is a soft $T_{2}$ space.

Lemma 2.11. Let $(X, \tau, E)$ be a finite soft $T_{2}$ space. Then $(X, \tau, E)$ is a soft discrete space.

Proof. Proof follows from theorem 2.9 and theorem 2.5

Lemma 2.12. If $(X, \tau, E)$ is a countable soft $T_{2}$ space and if every soft $G_{\delta}$ set is soft open in $(X, \tau, E)$, then $(X, \tau, E)$ is a soft discrete space.

Proof. Proof follows from theorem 2.9 and theorem 2.6 . 
Theorem 2.13. Let $(X, \tau, E)$ be a soft topological space. Then $(X, \tau, E)$ is a soft $T_{2}$ space if and only if for any soft points $x_{e_{i}}$ and $y_{e_{j}}$, there exist two soft closed neighbourhoods $(H, E)$ and $(K, E)$ containing disjoint soft open sets containing $x_{e_{i}}$ and $y_{e_{j}}$ respectively such that $(H, E) \tilde{\cup}(K, E)=\tilde{X}$.

Proof. Since $(X, \tau, E)$ is a soft $T_{2}$ space, for any two distinct soft points $x_{e_{i}}$ and $y_{e_{j}}$, there exist two soft open sets $(F, E)$ and $(G, E)$ such that $x_{e_{i}} \tilde{\epsilon}(F, E)$ and $y_{e_{j}} \tilde{\in}(G, E)$ such that $(F, E) \tilde{\cap}(G, E)=\tilde{\phi}$. Now $x_{e_{i}} \tilde{\in}\left(G^{c}, E\right), y_{e_{j}} \tilde{\epsilon}\left(F^{c}, E\right)$ and $\left(F^{c}, E\right) \tilde{\cup}\left(G^{c}, E\right)=\tilde{X}$. Note that $(F, E) \tilde{\subseteq}\left(G^{c}, E\right)$ and $(G, E) \tilde{\subseteq}\left(F^{c}, E\right)$. Let $\left(F^{c}, E\right)=(K, E)$ and $\left(G^{c}, E\right)=(H, E)$. Then we have two soft closed neighbourhoods $(H, E)$ and $(K, E)$ containing disjoint soft open sets $(F, E)$ and $(G, E)$ respectively, such that $x_{e_{i}} \tilde{\in}(F, E), y_{e_{j}} \tilde{\in}(G, E)(H, E) \tilde{\cup}(K, E)=\tilde{X}$.

Conversely let $x_{e_{i}}$ and $y_{e_{j}}$ be two distinct soft points. Then there exist two soft closed neighbourhoods $(H, E)$ and $(K, E)$ and two soft open sets $(L, E)$ containing $x_{e_{i}}$ and $(M, E)$ containing $y_{e_{j}}$ such that $(L, E) \tilde{\subseteq}(H, E),(M, E) \tilde{\subseteq}(K, E)$, $(L, E) \tilde{\cap}(M, E)=\tilde{\phi}$ and $(H, E) \tilde{\cup}(K, E)=\tilde{X}$. This proves that $(X, \tau, E)$ is a soft $T_{2}$ space

Theorem 2.14. Product of soft $T_{2}$-spaces is a soft $T_{2}$-space

Proof. Let $\left\{\left(X_{i}, \tau_{i}, E_{i}\right): i \in I\right\}$ be the collection of soft topoloogical spaces and $\left(\prod X_{i}, \prod \tau_{i}, \prod E_{i}\right)$ be their product soft toplogical space. Suppose $\mathbf{x}_{\mathbf{e}}$ and $\mathbf{y}_{\mathbf{f}}$ be two distinct soft points, where $\mathbf{x}=<x_{i}>_{i \in I}, \mathbf{y}=<y_{i}>_{i \in I} \quad x_{i}, y_{i} \in X_{i}$ and $\mathbf{e}=<e_{i}>_{i \in I}, \mathbf{f}=<f_{i}>_{i \in I}, e_{i}, f_{i} \in E_{i}$. Then there exists atleast one $\beta \in I$ such that $x_{\beta} \neq y_{\beta}$ or there exist $e_{i_{k}}, e_{i_{m}} \in E_{i}$ such that $e_{i_{k}} \neq e_{i_{m}}$.

Case: 1

If $x_{\beta} \neq y_{\beta},\left(p_{q}\right)_{\beta}\left(\mathbf{x}_{\mathbf{e}}\right)=\left(p_{\beta_{q_{\beta}}}\right)\left(\mathbf{x}_{\mathbf{e}}\right)=p_{\beta}(\mathbf{x})_{\mathbf{q}_{\beta}(\mathbf{e})}=x_{\beta_{e_{\beta}}}$ and $\left(p_{q}\right)_{\beta}\left(\mathbf{y}_{\mathbf{f}}\right)=\left(p_{\beta_{q_{\beta}}}\right)$ $\left(\mathbf{y}_{\mathbf{f}}\right)=p_{\beta}(\mathbf{y})_{\mathbf{q}_{\beta}(\mathbf{f})}=y_{\beta_{f_{\beta}}}$. Since $X_{\beta}$ is a soft $T_{2}$ space, there are disjoint soft open sets $\left(F_{\beta}, E_{\beta}\right)$ and $\left(G_{\beta}, E_{\beta}\right)$ such that $x_{\beta_{e_{\beta}}} \tilde{\in}\left(F_{\beta}, E_{\beta}\right)$ and $y_{\beta_{f_{\beta}}} \tilde{\in}\left(G_{\beta}, E_{\beta}\right)$. Then the subbasic members $\left(p_{q}\right)_{\beta}^{-1}\left(F_{\beta}, E_{\beta}\right)$ and $\left(p_{q}\right)_{\beta}^{-1}\left(G_{\beta}, E_{\beta}\right)$ are the soft open sets such that $\mathbf{x}_{\mathbf{e}} \tilde{\in}\left(p_{q}\right)_{\beta}^{-1}\left(F_{\beta}, E_{\beta}\right)$ and $\mathbf{y}_{\mathbf{f}} \tilde{\in}\left(p_{q}\right)_{\beta}^{-1}\left(G_{\beta}, E_{\beta}\right)$. Let $\mathbf{z}_{\mathbf{g}} \tilde{\in}\left(p_{q}\right)_{\beta}^{-1}\left(F_{\beta}\right.$, $\left.E_{\beta}\right) \tilde{\cap}\left(p_{q}\right)_{\beta}^{-1}\left(G_{\beta}, E_{\beta}\right)$, where $\mathbf{z}=<z_{i}>_{i \in I}, z_{i} \in X_{i}$ and $\mathbf{g}=<g_{i}>_{i \in I}, g_{i} \in E_{i}$. Then $\mathbf{z}_{\mathbf{g}} \tilde{\epsilon}\left(p_{q}\right)_{\beta}^{-1}\left(F_{\beta}, E_{\beta}\right)$ and $\mathbf{z}_{\mathbf{g}} \tilde{\in}\left(p_{q}\right)_{\beta}^{-1}\left(G_{\beta}, E_{\beta}\right) . \quad\left(p_{q}\right)_{\beta}\left(\mathbf{z}_{\mathbf{g}}\right) \tilde{\epsilon}\left(p_{q}\right)_{\beta}\left(\left(p_{q}\right)_{\beta}^{-1}\right.$ $\left.\left(F_{\beta}, E_{\beta}\right)\right)$ and $\left(p_{q}\right)_{\beta}\left(\mathbf{z}_{\mathbf{g}}\right) \tilde{\in}\left(p_{q}\right)_{\beta}\left(\left(p_{q}\right)_{\beta}^{-1}\left(G_{\beta}, E_{\beta}\right)\right)$. That is $z_{\beta_{g_{\beta}}} \tilde{\in}\left(F_{\beta}, E_{\beta}\right)$ and $z_{\beta_{g_{\beta}}} \tilde{\in}\left(G_{\beta}, E_{\beta}\right)$ which is a contradiction to our assumption of soft $T_{2}$ space.

\section{Case: 2}

If $e_{i_{k}} \neq e_{i_{m}}$, there are disjoint soft open sets $\left(F_{i_{k}}, E_{i}\right)$ and $\left(F_{i_{m}}, E_{i}\right)$ such that $x_{e_{i_{k}}}$ $\tilde{\epsilon}\left(F_{i_{k}}, E_{i}\right)$ and $x_{e_{i_{m}}}\left(=y_{e_{i_{m}}}\right) \tilde{\epsilon}\left(F_{i_{m}}, E_{i}\right)$. Then $\left(p_{q}\right)_{i}^{-1}\left(F_{i_{k}}, E_{i}\right)$ and $\left(p_{q}\right)_{i}^{-1}\left(F_{i_{m}}\right.$, $\left.E_{i}\right)$ are disjoint soft open sets containing $\mathbf{x}_{\mathbf{e}}$ and $\mathbf{y}_{\mathbf{f}}$ respectively. Let $\mathbf{z}_{\mathbf{g}} \tilde{\in}\left(p_{q}\right)_{i}^{-1}$ $\left(F_{i_{k}}, E_{i}\right) \tilde{\cap}\left(p_{q}\right)_{i}^{-1}\left(F_{i_{m}}, E_{i}\right)$, where $\mathbf{z}=<z_{i}>_{i \in I}, z_{i} \in X_{i}$ and $\mathbf{g}=<g_{i}>_{i \in I}, g_{i} \in E_{i}$. Then $\mathbf{z}_{\mathbf{g}} \tilde{\in}\left(p_{q}\right)_{i}^{-1}\left(F_{i_{k}}, E_{i}\right)$ and $\mathbf{z}_{\mathbf{g}} \tilde{\in}\left(p_{q}\right)_{i}^{-1}\left(F_{i_{m}}, E_{i}\right) . \quad\left(p_{q}\right)_{i}\left(\mathbf{z}_{\mathbf{g}}\right) \tilde{\in}\left(p_{q}\right)_{i}\left(\left(p_{q}\right)_{i}^{-1}\right.$ $\left.\left(F_{i_{k}}, E_{i}\right)\right)$ and $\left(p_{q}\right)_{i}\left(\mathbf{z}_{\mathbf{g}}\right) \tilde{\in}\left(p_{q}\right)_{i}\left(\left(p_{q}\right)_{i}^{-1}\left(F_{i_{m}}, E_{i}\right)\right)$. That is $z_{i_{g_{i}}} \tilde{\in}\left(F_{i_{k}}, E_{i}\right)$ and $z_{i_{g_{i}}}$ $\tilde{\epsilon}\left(F_{i_{m}}, E_{i}\right)$ which is a contradiction to our assumption of soft $T_{2}$ space.

Definition 2.7. Let $(X, \tau, E)$ be a soft topological space. Then $(X, \tau, E)$ is a soft Urysohn space or soft $T_{2 \frac{1}{2}}$ space if for any two soft points $x_{e_{i}}$ and $y_{e_{j}}$, there exist two soft open sets $(F, E)$ and $(G, E)$ such that $x_{e_{i}} \tilde{\in}(F, E), y_{e_{j}} \tilde{\in}(G, E)$ and $C l(F, E) \tilde{\cap} C l(G, E)=\tilde{\phi}$. 
Theorem 2.15. Every soft $T_{2 \frac{1}{2}}$-space is a soft $T_{2}$-space.

Proof. Proof is straight forward

Theorem 2.16. Soft subspace of soft $T_{2 \frac{1}{2}}$-space is a soft $T_{2 \frac{1}{2}}$-space.

Proof. Proof is similar to theorem 2.10

Theorem 2.17. Let $(X, \tau, E)$ be a soft topological space. Then $(X, \tau, E)$ is a soft $T_{2 \frac{1}{2}}$ space if and only if for any two soft points $x_{e_{i}}$ and $y_{e_{j}}$, there exist two soft open sets $(H, E)$ and $(K, E)$ such that $x_{e_{i}} \tilde{\in}(H, E), y_{e_{j}} \tilde{\in}(K, E)$ and $(H, E)$ and $(K, E)$ containing the disjoint closed soft neighbourhoods of $x_{e_{i}}$ and $y_{e_{j}}$ respectively with $(H, E) \tilde{\cup}(K, E)=\tilde{X}$.

Proof. Since $(X, \tau, E)$ be a soft $T_{2 \frac{1}{2}}$ space, for any soft points $x_{e_{i}}$ and $y_{e_{j}}$, there exist two soft open sets $(F, E)$ and $(G, E)$ such that $x_{e_{i}} \tilde{\in}(F, E)$ and $y_{e_{j}} \tilde{\in}(G, E)$ such that $C l(F, E) \tilde{\cap} C l(G, E)=\tilde{\phi}$. Now $x_{e_{i}} \tilde{e}[C l(G, E)]^{c}$ and $y_{e_{j}} \tilde{\epsilon}[C l(F, E)]^{c}$ and $[C l(F, E)]^{c} \tilde{\cup}[C l(G, E)]^{c}=\tilde{X}$. Note that $C l(F, E) \tilde{\subseteq}[C l(G, E)]^{c}$ and $C l(G, E) \tilde{\subseteq}$ $[C l(F, E)]^{c}$. Let $[C l(F, E)]^{c}=(K, E)$ and $[C l(G, E)]^{c}=(H, E)$. Then we have two soft open sets $(H, E)$ and $(K, E)$ containing $x_{e_{i}}$ and $y_{e_{j}}$ respectively, such that $x_{e_{i}} \tilde{\epsilon}(F, E) \tilde{\subseteq} C l(F, E) \tilde{\subseteq}(H, E), y_{e_{j}} \tilde{\epsilon}(G, E) \tilde{\subseteq} C l(G, E) \tilde{\subseteq}(K, E)$ and $(H, E) \tilde{\cup}(K, E)=\tilde{X}$. Thus $(H, E)$ and $(K, E)$ are soft open sets containing the disjoint closed neighbourhoods $C l(F, E)$ and $C l(G, E)$, respectively such that $x_{e_{i}} \tilde{\in} C l(F, E), y_{e_{j}} \tilde{\in} C l(G, E)$ and $(H, E) \tilde{\cup}(K, E)=\tilde{X}$.

Conversely, let $x_{e_{i}}$ and $y_{e_{j}}$ be two distinct soft points. By our assumption, there exist two soft open sets $(H, E)$ and $(K, E)$ containing disjoint closed neighbourhoods $(L, E)$ and $(M, E)$ of $x_{e_{i}}$ and $y_{e_{j}}$ respectively such that $(H, E) \tilde{\cup}(K, E)=\tilde{X}$. Note that there are soft open sets $(F, E)$ and $(G, E)$ such that $(F, E) \tilde{\subseteq} C l(F, E) \tilde{\subseteq}(L, E) \tilde{\subseteq}$ $(H, E),(G, E) \tilde{\simeq} C l(G, E) \tilde{\subseteq}(M, E) \tilde{\subseteq}(K, E)$ and $(L, E) \tilde{\cap}(M, E)=\tilde{\phi}$. So that $C l(F, E) \tilde{\cap}$ $C l(G, E)=\tilde{\phi}$. That is $(F, E)$ and $(G, E)$ are soft open sets containing $x_{e_{i}}$ and $y_{e_{j}}$ such that $C l(F, E) \tilde{\cap} C l(G, E)=\tilde{\phi}$. Thus $(X, \tau, E)$ is a soft $T_{2 \frac{1}{2}}$ space.

Soft single point space discussed in [5] is not a soft $T_{0}$ or $T_{1}$ or $T_{2}$ or $T_{2 \frac{1}{2}}$ space. Because for the soft points $x_{e_{i}}$ and $x_{e_{j}}$, there is no soft open set containing $x_{e_{i}}$ not containing $x_{e_{j}}$.

Theorem 2.18. Product of soft $T_{2 \frac{1}{2}}$-spaces is a soft $T_{2 \frac{1}{2}}$-space

Proof. Proof is similar to theorem 2.14

Lemma 2.19. Let $(X, \tau, E)$ be a finite soft $T_{2 \frac{1}{2}}$ space. Then $(X, \tau, E)$ is a soft discrete space.

Proof. Proof follows from theorem 2.15, theorem 2.9 and theorem 2.5 .

Lemma 2.20. If $(X, \tau, E)$ is a countable soft $T_{2 \frac{1}{2}}$ space and if every soft $G_{\delta}$ set is soft open in $(X, \tau, E)$, then $(X, \tau, E)$ is a soft discrete space.

Proof. Proof follows from theorem 2.15, theorem 2.9 and theorem 2.6 . 


\section{Conclusion}

For the soft separation axioms of soft points defined on soft topological space, we discuss the characterizations and properties of soft $T_{0}, T_{1}, T_{2}$ and soft $T_{2 \frac{1}{2}}$ spaces. Also it is verified that the product of soft $T_{i}$ spaces, $i=1,2,2 \frac{1}{2}$ is a soft $T_{i}$ space. But there is an example given here for the product of soft $T_{0}$ spaces need not be a soft $T_{0}$ space. Also we provide correct examples for the wrong examples example:1, example:2 and example:3 given in article [8.

Acknowledgments. The authors are grateful to the anonymous reviewers and the editor for their valuable suggestions and useful comments to improve the manuscript.

\section{REFERENCES}

[1] H. Aktas and N. Cagman, Soft sets and soft groups, Inf. Sci., 177(13) (2007) 2726 - 2735.

[2] A. Aygunoglu and H. Aygun, Some notes on soft topological spaces, Neural Comput. Applic., 21(1) (2012) 113 - 119.

[3] K.V. Babitha and J.J. Sunil, Soft set relations and functions, Comput. Math. Appl., 60 (2010) 1840 - 1849.

[4] D.N. Georgiou and A.C. Megaritis, Soft set theory and topology, Appl. Gen. Topol., 15(1) (2014) 93 - 109.

[5] O. Gocur and A. Kopuzlu, Some new properties of soft separation axioms, Ann. Fuzzy Math. Inform., 9(3) (2015) 421 - 429.

[6] C. Gunduz Aras, A. Sonmez and H. Cakalli, An approach to soft functions, J. Math. Anal., 8(2) (2017) 129-138.

[7] S. Hussain and B. Ahmad, Some properties of soft topological spaces, Comput. Math. Appl., 62(11) (2011) 4058 - 4067.

[8] S. Hussain and B. Ahmad, Soft separation axioms in soft topological spaces, Hacet. J. Math. Stat., 44(3) (2015) 559 - 568.

[9] A. Kharral and B. Ahmad, Mappings on soft classes, New Math. Nat. Comput., 7(3) (2011) $471-481$.

[10] P.K. Maji, R. Biswas and A.R. Roy, Soft set theory, Comput. Math. Appl., 45 (2003) 555 562.

[11] P.K. Maji, A.R. Roy and R. Biswas, An application of soft sets in a decision making problems, Comput. Math. Appl., 44 (2002) 1077 - 1083.

[12] D. Molodtsov, Soft set theory - First results, Comput. Math. Appl., 37, (2019) 19 - 31.

[13] S.K. Nazmul and S.K. Samanta, Neighbourhood properties of soft topological spaces, Ann. Fuzzy Math. Inform., 6(1) (2013) 1 - 15.

[14] T.Y. Ozturk and S. Bayramov, Soft mapping spaces, The Scientific World Journal, Art. ID.: $307292(2014) 1-8$.

[15] J.R. Porter and R. Grandwoods, Extensions and absolutes of Hausdorff spaces, SpringerVerlag, New york (1988).

[16] M. Shabir and M. Naz, On soft topological spaces, Comput. Math. Appl., 61 (2011) 1786 1799.

[17] A. Singh and N.S. Noorie, Remarks on soft axioms, Ann. Fuzzy Math. Inform., 14(5) (2017) $503-513$.

[18] O. Tantawy, S.A. El-Sheikh and S. Hamde, Seperation axioms on soft topological spaces, Ann. Fuzzy Math. Inform., 11(4) (2016) 511 - 525.

[19] B.P. Varol and H. AygAun, On soft Hausdorff spaces, Ann. Fuzzy Math. Inform., 5(1) (2013) $15-24$.

[20] D. Wardowski, On a soft mapping and its fixed points, Fixed Point Theory Appl., 182 (2013) $1-11$.

[21] I. Zorlutuna, M.Akdag, W.K. Min and S. Atmaca, Remarks on soft topological spaces, Ann. Fuzzy Math. Inform., 3(2) (2012) 171 - 185. 
SOFT SEPARATION AXIOMS AND SOFT PRODUCT OF SOFT TOPOLOGICAL SPACES 75

Ramkumar Solai,

Government Polytechnic College, Gandharvakottai, Pudukkottai district - 613301, TAMILNADU, INDiA.

Email address: ramkumarsolai@gmail.com, ORCID: https://orcid.org/0000-0001-5011-774X

Vinoth SUBbiah,

Vignan's University, Vadlamudi, Gundur - 522 213, Andhra Pradhesh, India.

Email address: vinosugan2569@gmail.com, ORCID: https://orcid.org/0000-0001-8621-8032 